\title{
Reducing hydroelastic response of very large floating structures by altering their plan shapes
}

\author{
Z.Y. Tay* and C.M. Wang \\ Department of Civil and Environmental Engineering, National University of Singapore, \\ Kent Ridge, Singapore, 119260 \\ (Received December 2, 2011, Revised February 29, 2012, Accepted March 7, 2012)
}

\begin{abstract}
Presented herein is a study on reducing the hydroelastic response of very large floating structures (VLFS) by altering their plan shapes. Two different categories of VLFS geometries are considered. The first category comprises longish VLFSs with different fore/aft end shapes but keeping their aspect ratios constant. The second category comprises various polygonal VLFS plan shapes that are confined within a square boundary or a circle. For the hydroelastic analysis, the water is modeled as an ideal fluid and its motion is assumed to be irrotational so that a velocity potential exists. The VLFS is modeled as a plate by adopting the Mindlin plate theory. The VLFS is assumed to be placed in a channel or river so that only the head sea condition is considered. The results show that the hydroleastic response of the VLFS could be significantly reduced by altering its plan shape.
\end{abstract}

Keywords: very large floating structure (VLFS); geometries; arbitrary shapes; mitigation methods; hydroelastic response

\section{Introduction}

Very large floating structure (VLFS) has been proposed as an alternative to the traditional land reclamation solution in creating land parcels from the sea. Owing to their large surface areas and relatively smaller depths, VLFS behaves elastically under wave action. Therefore, it is important to mitigate the hydroelastic responses of the floating structures for applications that demands stringent serviceability requirements.

There are several ways in mitigating the hydroelastic response of the VLFS. The conventional method is to install bottom founded breakwaters such as those used for the Mega-float (Suzuki 2005) and floating oil storage bases in Shirashima and Kamigoto Islands (Wang et al. 2008). The bottom founded breakwater is the most effective means in attenuating the wave forces (Wang et al. 2010). However, such type of breakwater is difficult to construct, expensive and not environmentally friendly as they damage the seabed and obstruct the current flow. Alternatively, the more environmentally friendly floating breakwater could effectively attenuate the wave forces acting on the VLFS. Such a floating breakwater is proposed for the floating fuel storage facility in Singapore (Tay et al. 2009).

Researchers such as Ohta et al. (1999) and Watanabe et al. (2003a,b) later proposed anti-motion

*Corresponding author, Dr., E-mail: ceetzy@nus.edu.sg 
devices by attaching vertical or horizontal plates to the fore end of the VLFS. These horizontal plates help in generating reverse flow at the aft-end of the plate that counteract with the incident wave. Curtain wall with slits (Masanobu et al. 2003) attached to the fore-end of VLFS have also been proposed to mitigate the hydroelastic response through eddies generated by the slits.

Pinkster (1997) also borrowed the air-cushion technology used in hovercrafts and surface effect ships (SES) to reduce the hydroelastic response of VLFS. The air-cushion comprises vertical walls submerged in the water so that the hull of the VLFS is raised above the sea water. The air between the hull and seawater is thus compressed and this helps in dampening the hydroelastic response of the VLFS such as in floating airports when airplanes takeoff or land.

Khabakhpasheva and Korobkin (2002) proposed to connect modules in VLFS by using hinge connector in order to minimize the hydroelastic response of the VLFS. Wang et al. (2009) also investigated the effect of semi-rigid connectors in mitigating the hydroelastic response of a VLFS modeled as a beam. Gao et al. (2011) then extended the beam model to a plate model and found that the line-hinge connector is able to reduce the hydroelastic response of the VLFS significantly. Wang et al. (2012) later found that the hydroelastic response of the VLFS with hinge connectors could be further reduced by introducing gill cells in location where the structure experiences maximum deflection.

In a paper by Kim et al. (2005b), hydroelastic response is found to be reduced when an articulated plate with different stiffnesses and shapes are attached to the fore-end of the VLFS by using hinge connector. The mitigation method by increasing the stiffness is not preferable as it increases the material, hence the cost of the VLFS. In the literature, not many research studies have been conducted on mitigating hydroelastic response by altering the shapes of the VLFS. Hence, this prompted us to investigate the effect of different geometries with the goal to minimize the hydroelastic response of a VLFS that is modeled as a continuous plate.

\section{Mathematical formulation}

\subsection{Plate-water model}

Consider a VLFS with an arbitrary shape. It has a structural length $L$ and height $h$ and it is assumed to be of zero draft. The VLFS is modeled as a floating plate and assumed to be perfectly flat with free edges. The water is assumed to be an ideal fluid (inviscid and incompressible) and its flow is irrotational. Fig. 1 shows the schematic diagram of the coupled water-plate problem. The water domain is denoted by $\Omega$. The symbols $\boldsymbol{S}_{\boldsymbol{H} B}, \boldsymbol{S}_{\boldsymbol{F}}$ and $\boldsymbol{S}_{S B}$ represent the plate domain, the free water surface and the seabed, respectively. The water motion and plate deflection are assumed to be in a steady state harmonic motion with a circular frequency $\omega$. The free and undisturbed water surface is at $z=0$ while the seabed is found at $z=-H$. An incident wave $\phi_{I n}$ of period $T$ and wave height $2 A$ enters the computational domain at a wave angle $\theta$ with wavelength-to-structure length ratio $\alpha=\lambda L$. The deflection $w$ is measured from the free and undisturbed water surface.

\subsection{Equations of motion for Mindlin plate}

The Mindlin (thick) plate theory is used to model the VLFS. The plate material is assumed to be isotropic and obeys Hooke's law. For simplicity, the zero draft assumption is made. The motion of 


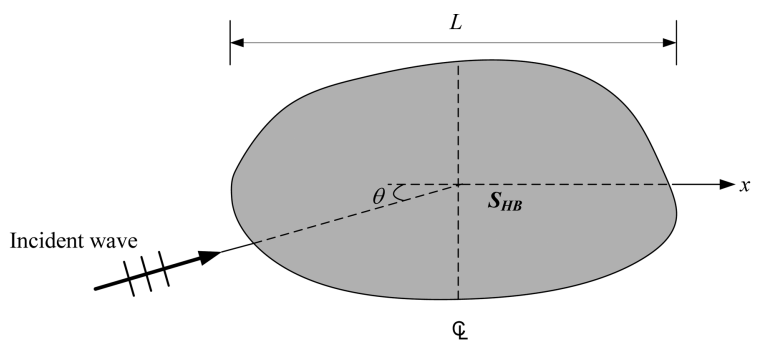

(a) Plan view

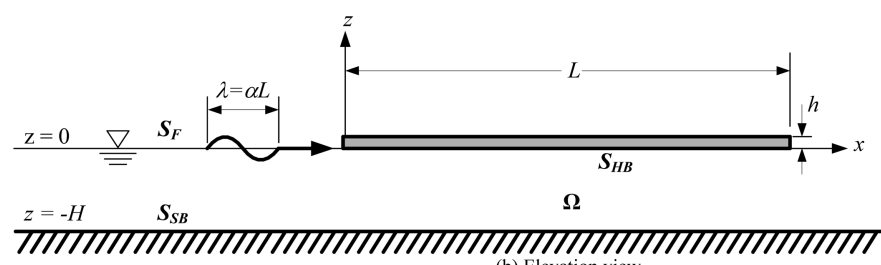

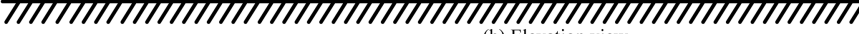

Fig. 1 Schematic diagram of coupled water-plate problem (a) Plan view and (b) side view

the Mindlin plate is represented by the vertical displacement $w(x, y)$, the rotation $\Psi_{x}(x, y)$ about the $y$-axis and the rotation $\Psi_{y}(x, y)$ about the $x$-axis. The governing equations of motion for the Mindlin plate (after omitting the time factor $e^{-i \omega t}$ ) are (Liew et al. 1998)

$$
\begin{gathered}
\kappa^{2} G h\left[\left(\frac{\partial^{2} w}{\partial x^{2}}+\frac{\partial^{2} w}{\partial y^{2}}\right)+\left(\frac{\partial \Psi_{x}}{\partial x}+\frac{\partial \Psi_{y}}{\partial y}\right)\right]+\rho_{p} h \omega^{2} w=p(x, y) \\
D\left[\frac{(1-v)}{2}\left(\frac{\partial^{2} \Psi_{x}}{\partial x^{2}}+\frac{\partial^{2} \Psi_{x}}{\partial y^{2}}\right)+\frac{(1+v)}{2}\left(\frac{\partial^{2} \Psi_{x}}{\partial x^{2}}+\frac{\partial^{2} \Psi_{x}}{\partial x \partial y}\right)\right]-\kappa^{2} G h\left(\frac{\partial w}{\partial x}+\Psi_{x}\right)+\rho_{p} \frac{h^{3}}{12} \omega^{2} \Psi_{x}=0 \\
D\left[\frac{(1-v)}{2}\left(\frac{\partial^{2} \Psi_{y}}{\partial y^{2}}+\frac{\partial^{2} \Psi_{y}}{\partial x^{2}}\right)+\frac{(1+v)}{2}\left(\frac{\partial^{2} \Psi_{y}}{\partial y^{2}}+\frac{\partial^{2} \Psi_{x}}{\partial x \partial y}\right)\right]-\kappa^{2} G h\left(\frac{\partial w}{\partial y}+\Psi_{y}\right)+\rho_{p} \frac{h^{3}}{12} \omega^{2} \Psi_{y}=0
\end{gathered}
$$

where $G=E /[2(1+v)]$ is the shear modulus, $\kappa^{2}$ the shear correction factor taken as $5 / 6, \rho_{p}$ the mass density of the plate, $h$ the thickness of the plate, $G=E h^{3} /\left[2\left(1+v^{2}\right)\right]$ the flexural rigidity, $E$ the Young's modulus and $v$ the Poisson ratio. The pressure $p(x, y)$ in Eq. (1) comprises the hydrostatic and hydrodynamic pressure, i.e.

$$
p(x, y)=\rho g w+i \omega p \phi(x, y, 0) \text { on } \boldsymbol{S}_{\boldsymbol{H} \boldsymbol{B}}
$$

where $\rho$ is the mass density of water, $g$ the gravitational acceleration and $\phi(x, y, 0)$ the velocity potential of water. The boundary conditions at the free edges of the floating plate are (Liew et al. 1998) 


$$
\begin{gathered}
\text { Bending moment } M_{n m}=D\left[\frac{\partial \Psi_{n}}{\partial n}+v \frac{\partial \Psi_{s}}{\partial s}\right]=0 \\
\text { Twisting moment } M_{n s}=D\left(\frac{1-v}{2}\right)\left[\frac{\partial \Psi_{n}}{\partial s}+\frac{\partial \Psi_{s}}{\partial n}\right]=0 \\
\text { Shear force } Q_{n}=\kappa^{2} G h\left[\frac{\partial w}{\partial n}+\Psi_{n}\right]=0
\end{gathered}
$$

where $s$ and $n$ denote the tangential and normal directions to the section of the plate.

\subsection{Equations of motion for water}

Water is assumed to be an ideal fluid and has an irrotational flow so that a velocity potential exists. Thus the single frequency velocity potential of water must satisfy the Laplace's equation (Sarpkaya and Isaacson 1981)

$$
\nabla^{2} \phi(x, y, z)=0 \text { in } \Omega
$$

and the boundary conditions

$$
\begin{gathered}
\frac{\partial \phi}{\partial z}(x, y, 0)=-i \omega w(x, y) \text { on } \boldsymbol{S}_{\boldsymbol{H} \boldsymbol{B}} \\
\frac{\partial \phi}{\partial z}(x, y, 0)=\frac{\omega^{2}}{g} \phi(x, y) \text { on } \boldsymbol{S}_{\boldsymbol{F}} \\
\frac{\partial \phi}{\partial z}(x, y,-H)=0 \text { on } \boldsymbol{S}_{\boldsymbol{S B}}
\end{gathered}
$$

Eq. (7) implies that no gap exists between the plate and the water-free surface whereas Eq. (8) is derived from the linearized Bernoulli equation where the pressure is taken as zero at the water surface. Eq. (10) is the boundary condition at the seabed which expresses impermeability, i.e., no fluid enters or leaves the seabed and hence the velocity component normal to the seabed is zero.

The wave velocity potential must also satisfy the Sommerfeld radiation condition as $|\mathbf{x}| \rightarrow \infty$ (Faltinsen 1990)

$$
\lim _{|x| \rightarrow \infty} \sqrt{\mid \mathbf{x}}\left(\frac{\partial}{\partial|\mathbf{x}|}-i k\right)\left(\phi-\phi_{I n}\right)=0 \text { on } \boldsymbol{S}_{\infty}
$$

where $\mathbf{x}=(x, y), \phi_{I n}$ is the incident velocity potential and $\omega$ the wave frequency. $\boldsymbol{S}_{\infty}$ represents the artificial fluid boundary at infinity.

\subsection{Modal expansion of plate motion and water potential}

The plate Eq. (1) indicates that the response of the plate $w(x, y)$ is coupled with the fluid motions or velocity potential $\phi(x, y, z)$. On the other hand, the fluid motion can only be obtained when the 
plate deflection $w(x, y)$ is specified in the boundary condition (7) for the fluid part. In order to decouple this interaction problem into a hydrodynamic problem in terms of the velocity potential and a plate vibration problem in terms of the generalized displacement, we adopt the modal expansion method as proposed by Newman (1994). In this method, the deflection of the plate $w(x$, $y$ ) is expanded by $N$ series of products of the modal function $c_{l}^{w}(x, y)$ and complex amplitudes $\varsigma_{l}$

$$
w(x, y)=\sum_{l=1}^{N} \zeta_{l} c_{l}^{w}(x, y)
$$

The single frequency velocity potential $\phi$ of water can be separated into the diffracted part $\phi_{D}$ and the radiated part $\phi_{R}$ based on the linear potential theory. The radiated potential $\phi_{R}$ can be further decomposed as (Eatock Taylor and Waite 1978)

$$
\phi(x, y, z)=\phi_{D}(x, y, z)+\phi_{R}(x, y, z)=\phi_{D}(x, y, z)+\sum_{l=1}^{N} \zeta_{l} \phi_{l}(x, y, z)
$$

where $\phi_{l}(x, y, z)$ is the radiation potential corresponding to the unit-amplitude motion of the $l$-th modal function and $\zeta_{l}$ is the complex amplitudes which is assumed to be the same as those given in Eq. (11) (Newman 1994). The diffracted potential $\phi_{D}$ is taken as the sum of the incident potential $\phi_{I n}$ and the scattered potential $\phi_{S}$, where the incident velocity potential $\phi_{I n}$ is given by Eq. (14) and the scattered potential $\phi_{S}$ represents the outgoing wave from the body.

$$
\phi_{I n}=\frac{g A}{\omega} \frac{\cosh (k(z+H))}{\cosh k H} e^{i k(x \cos \theta+y \cos \theta)}
$$

where $\phi_{I n}$ is the incident plane wave, $\theta$ the incident angle, $k$ the wave number (related to the wave length $\lambda$ by $\mathrm{k}=2 \pi / \lambda$ ) and $\omega$ the wave frequency.

\subsection{Hydrodynamic analysis}

We substitute the expanded plate deflection and velocity potential (Eqs. (11) and (12)) into the Laplace's Eq. (6) and boundary conditions (Eqs. (7) to (10)) in order to obtain the Laplace's equation and boundary conditions for water in the decoupled form, i.e.

$$
\begin{gathered}
\nabla^{2} \phi_{l}(x, y, z)=0 \text { in } \Omega \\
\frac{\partial \phi_{l}}{\partial z}(x, y, 0)=\left\{\begin{array}{cl}
-i \omega c_{l}^{w}(x, y) & \text { for } l=1,2, \ldots, N \text { on } \boldsymbol{S}_{\boldsymbol{H} \boldsymbol{B}} \\
0 & \text { for } l=D
\end{array}\right. \\
\frac{\partial \phi_{l}}{\partial z}(x, y, 0)=\frac{\omega^{2}}{g} \phi_{l}(x, y, 0) \text { on } \boldsymbol{S}_{F} \\
\frac{\partial \phi_{l}}{\partial z}(x, y,-H)=0 \text { on } \boldsymbol{S}_{\boldsymbol{S B}}
\end{gathered}
$$




$$
\lim _{|x| \rightarrow \infty} \sqrt{|\mathbf{x}|}\left(\frac{\partial \phi_{l}}{\partial|\mathbf{x}|}-i k \phi_{l}\right)=0 \quad \begin{aligned}
& \text { for } l=1,2, \ldots, N \text { on } \boldsymbol{S}_{\infty} \\
& \text { for } l=S
\end{aligned}
$$

We then transform Eqs. (14) to (18) into an integral equation by using the Green's second identity (Wang et al. 2008).The resulting boundary integral equation is

$$
\phi_{l}(\mathbf{x})+\int_{S_{H B}} \frac{\partial G(\mathbf{x}, \xi)}{\partial z} \phi_{l}(\xi) d S_{\xi}=\int_{S_{H B}} G(\mathbf{x}, \xi) \frac{\partial \phi_{l}(\xi)}{\partial z} d S_{\xi}
$$

where $\mathbf{x}=(x, y)$ is the field point and $\xi=(\xi, \eta)$ the source point. $G(\mathbf{x}, \xi)$ is a free-surface Green's function for water of finite depth that satisfies the sea bed boundary condition, water free surface boundary condition and boundary at infinity (Linton 1999).

By further introducing the boundary conditions for $\partial \phi_{l} / \partial z$ into Eq. (19), Wang et al. (2008) obtained

$$
\phi_{l}(\mathbf{x})+\int_{S_{H B}} \frac{\partial G(\mathbf{x}, \xi)}{\partial z} \phi_{l}(\xi) d S_{\xi}=\left\{\begin{array}{cl}
\int_{S_{H B}}-i \omega G(\mathbf{x}, \xi) c_{l}^{w}(\xi) d S_{\xi} & \text { for } l=1,2, \ldots, N \\
4 \pi \phi_{I n} & \text { for } l=D
\end{array}\right.
$$

\subsection{Solution for plate and water motion}

The plate Eqs. (1) to (3) is solved by using the finite element (FE) method whereas the water Eq. (20) is solved using the boundary element (BE) method. In order to implement the hybrid FE-BE method, we need to obtain the discretized version of the plate and water equation. The element used for the finite element analysis of the arbitrary floating plate is the four-node quadrilateral thick plate element whereas the water domain is solved by using the constant panel method (Brebbia 1984).

The solution for the plate and water motion is determined according to the numerical scheme given in Tay et al. (2009).

\section{Problem definition}

The VLFS is assumed to be located in a channel/river, such that the waves only approach in a head sea direction, i.e., $\theta=0$ (see Fig. 1). The reflected waves due to the river bank is assumed to be very small and shall be ignored in this analysis. The floating structure is assumed to be continuous with the plate properties and sea states given in Table 1.

Various geometries of VLFS classified into two different categories are considered, i.e.

(I) Longish VLFS with various fore- and aft-ends shapes

(II) VLFS with various shapes confined in a same square boundary

The shapes considered in Categories I and II are given in Figs. 2 and 3, respectively. In Category I (Fig. 2), six longish VLFSs with length $L=300 \mathrm{~m}$, width $W=60 \mathrm{~m}$ and thickness of $2 \mathrm{~m}$ are considered. The shapes of the fore- and aft-ends of the VLFS are altered in order to investigate the effect of different shapes in reducing the hydroelastic response. Four different fore- and aft-end shapes, i.e., semi-circular, semi-decagonal, semi-hexagonal and triangular shape, are considered in 
Table 1 Plate properties and sea states

\begin{tabular}{cccc}
\hline \hline Parameter & Symbol & Unit & Value \\
\hline Plate mass density & $\rho$ & $\mathrm{kg} / \mathrm{m}^{3}$ & 256.25 \\
Young's modulus & $E$ & $\mathrm{GN} / \mathrm{m}^{2}$ & 11.9 \\
Poisson's ratio & $v$ & & 0.13 \\
Water depth & $H$ & $\mathrm{~m}$ & 58.5 \\
Wavelength-to-structure length ratio & $\alpha$ & & 0.2 to 1.0 \\
\hline
\end{tabular}

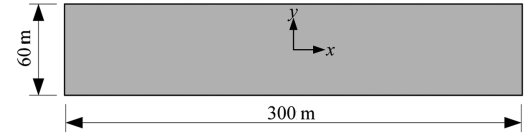

(a) Rectangular shape VLFS

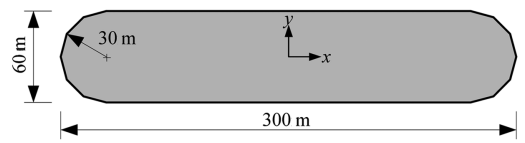

(c) Rectangular shape VLFS with semidecagonal ends

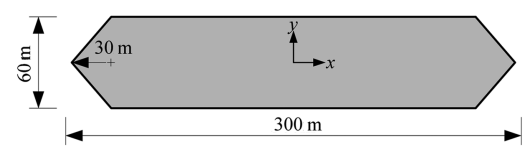

(e) Rectangular shape VLFS with triangular ends

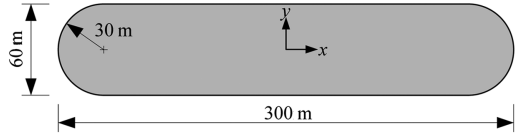

(b) Rectangular shape VLFS with semicircular ends

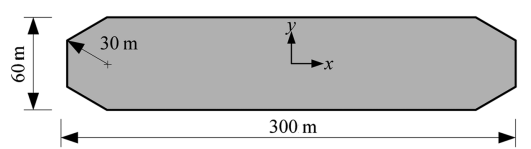

(d) Rectangular shape VLFS with semihexagonal ends

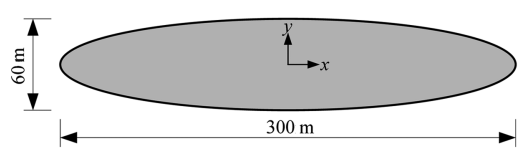

(f) Elliptical shape VLFS

Fig. 2 Longish VLFS with various fore- and aft-ends shapes



(a) Square

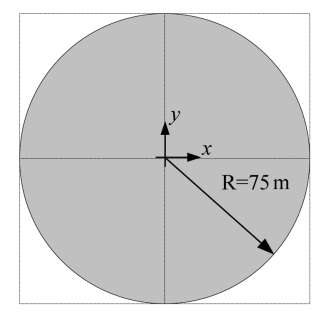

(b) Circular shape

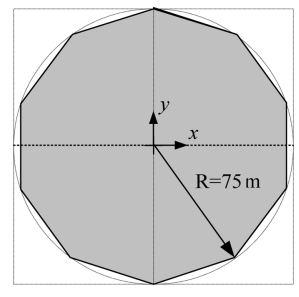

(c) Decagonal shape

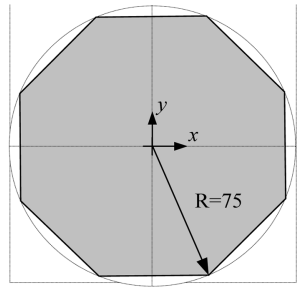

(d) Octagonal shape

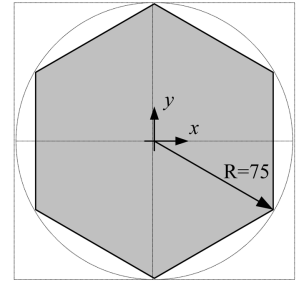

(e) Hexagonal shape

Fig. 3 Polygonal shaped VLFS 
the investigation, with each shape having a length of $30 \mathrm{~m}$ (see Fig. 2). An elliptical VLFS with a major axis of $150 \mathrm{~m}$ and a minor axis of $30 \mathrm{~m}$ (Fig. 2(f)) is also considered to represent VLFS with a streamline shape.

In Category II (Fig. 3), a square shape VLFS (i.e., plate with aspect ratio of 1.0) with a length of $150 \mathrm{~m}$ and thickness of $2 \mathrm{~m}$ is first considered. In order to investigate the effect of various shapes in reducing the hydroelastic response, the corners of the square is being replaced by various shapes such that VLFS with polygonal shapes with a radius of $75 \mathrm{~m}$ are formed (see Fig. 3). The polygons considered in the investigation are circular, decagon, octagon and hexagon.

The finite element meshes of the various VLFS shapes are modeled using the finite element software ABAQUS. Based on the convergence tests, it is found that the size of the four-node quadrilateral thick plate element and the constant fluid panel must be smaller than $\lambda / 10$ for accurate prediction of the hydroelastic deflection, where $\lambda$ is the wavelength. The results for the convergence tests and validation of the numerical scheme have been published in papers by Tay et al. (2009) and Gao et. al. (2011), and hence they will not be shown here.

\section{Results and discussions}

\subsection{Longish VLFS with various fore- and aft-ends shapes}

The hydroelastic responses of various longish VLFSs (Category I, see Fig. 2) subjected to a head sea condition are shown in Fig. 4. The wavelengths to structural length ratio considered in the investigation are $\alpha=0.2$ to 1.0. Fig. 4 shows that the VLFS with an elliptical shape is most effective in reducing the hydroelastic response of the VLFS followed by the VLFS with triangular ends. The elliptical shaped VLFS has the smallest response as it induces a greater scattering wave. Other fore-end shapes i.e., circular, semi-decagon and semi-octagon are also effective in mitigating the hydroelastic response as these fore-end shapes allow a smoother flow of water along the floating body. However, these various fore- and aft-end shapes are only effective when the wavelength to structure length ratio $\alpha$ is small, i.e., $\leq 0.3$. When $\alpha>0.3$, the hydroelastic response of the VLFS with various polygonal shapes starts to increase as compared to the rectangular shape VLFS. This may be due to the fact that the response of the VLFS is dominated by inertia force as the $\alpha$ increases, and thus the response of the rectangular shape VLFS with a larger mass (as compared to VLFS with other shapes) is the smallest.

\subsection{VLFS with various shapes confined in a same square boundary}

The hydroelastic response of various polygonal shapes VLFS (Category II, see Fig. 3) under head sea condition is investigated. In order to quantify the effectiveness of the flexible line connector on the hydroelastic response, we introduce the non-dimensional compliance parameter $\chi$, which is defined as

$$
\chi=\int_{S_{H B}}|w| d S_{H B}
$$





(a) $\lambda / L=0.2$

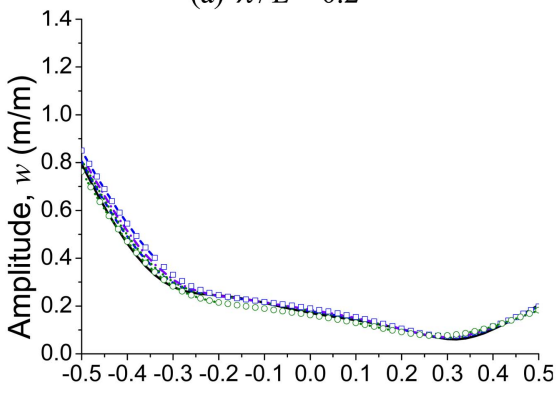

(b) $\lambda / L=0.3$

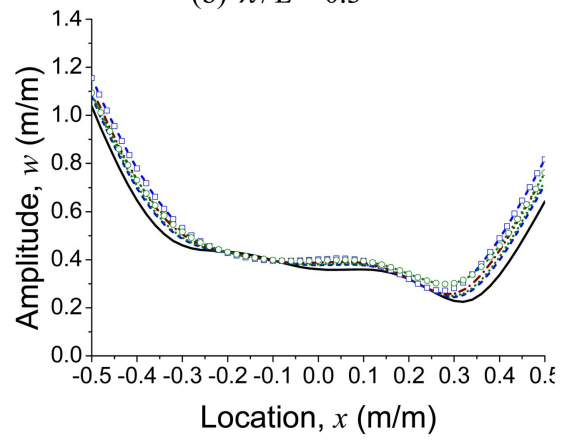

Location, $x(\mathrm{~m} / \mathrm{r}$
(c) $\lambda / L=0.4$

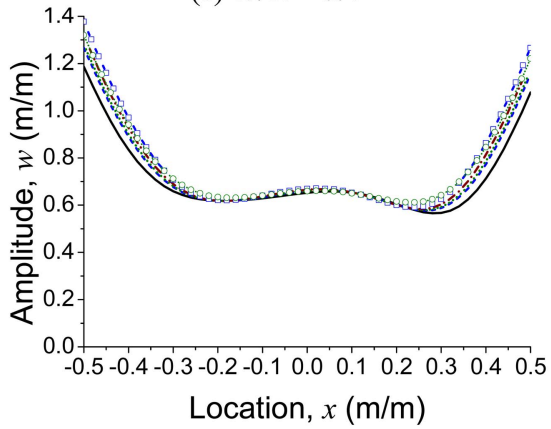

(d) $\lambda / L=0.6$

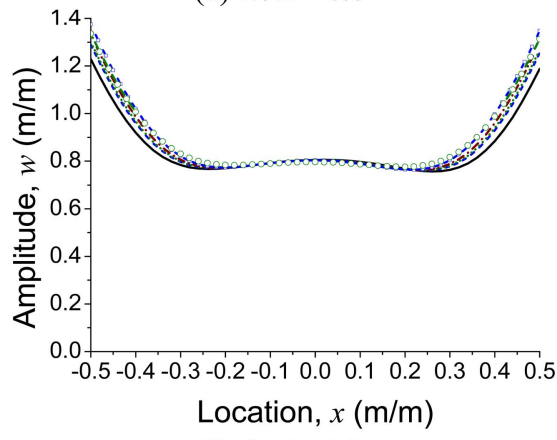

(e) $\lambda / L=0.8$

(f) $\lambda / L=1.0$

Fig. 4 Hydroelastic response of longish VLFS

A minimum value of the compliance parameter is desirable as it is tantamount to having a stiffer plate against wave action.

Fig. 5 presents the variation of the compliance $\chi$ with respect to different shapes (as given in Fig. 3) and wavelengths $\alpha$. It is shown that the hydroelastic response of the square VLFS is the largest and could be reduced by replacing its corners with polygonal shapes. It is found that the VLFS with hexagonal shape is the most effective in minimizing the hydroelastic response of the VLFS. This is because the hydroelastic response of the circular/polygonal plates is governed by the bending action coupled in both the $x$ and $y$-directions, whereas the hydroelastic responses of a longish VLFS (as discuss in the previous section) or a square VLFS under head sea condition are governed only by the bending action in one direction. Hence, the hydroelastic response of the VLFS considered in Fig. 3 could be further reduced if the structures are oriented in such a way that the vertex/corner of the polygon is pointing to the approaching waves (see Fig. 6). For example, by orientating the 




Fig. 5 Variation of compliances with respect to various shapes and wavelengths

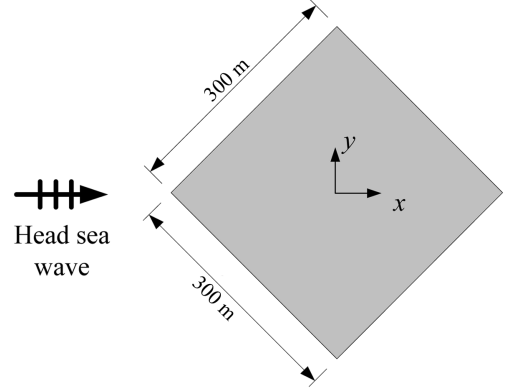

(a) Decagonal

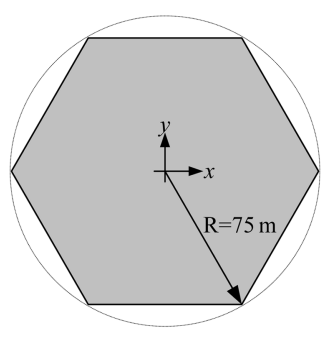

(b) Hexagonal

Fig. 6 Polygonal plate oriented such that corner/vertex points to approaching wave

square and hexagonal shape VLFS as shown in Fig. 6, the compliances are found to reduce as shown in Fig. 7. In fact, at a small wave length, i.e., $\alpha=0.3$, the hydroelastic response is the smallest for the square VLFS at this orientation. This is because the hexagonal with vertex pointing to the approaching waves would allow for a greater scattering wave. The effect of orientating the hexagonal shape VLFS vanishes as the wavelength is large due to the same argument as described in the previous section. It is noted that the effect of orientating the decagonal or octagonal shape VLFS in reducing the hydroelastic response is minimal as these shapes are approaching the shape of a circle (The hydroelastic response of wave approaching a circular shape is the same in all directions).

Fig. 8 shows the comparison of hydroelastic response between a square, a circular and polygonal VLFSs under a wavelength to structural length ratio $\alpha$ of 0.3 . It can be seen clearly that the VLFS with longer edge facing the approaching wave would experience higher deformations as compare to their counterpart with corners or rounded edge (for circular case) pointing to the approaching waves.

\section{Conclusions}

The effect of altering the plan shapes of VLFS on the hydroelastic response was investigated 


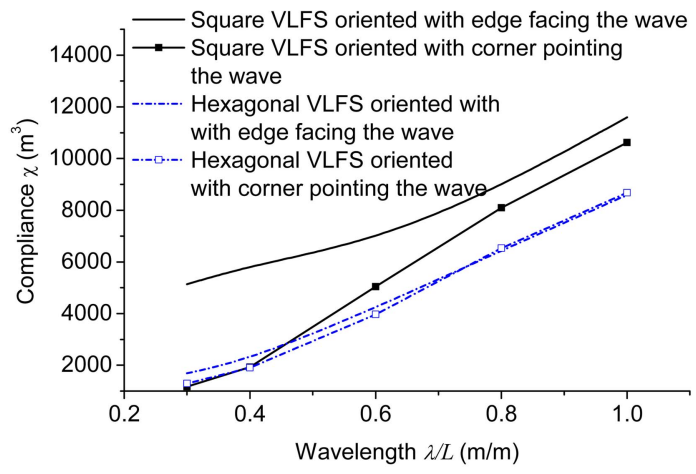

Fig. 7 Variation of compliances with respect to wavelength and polygonal shape VLFS with different orientations

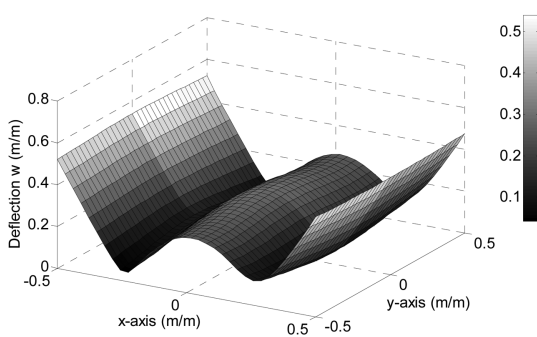

(a) Square VLFS

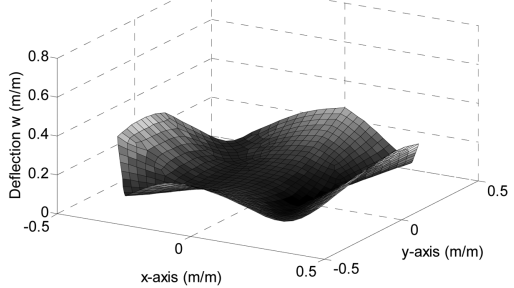

(c) Decagonal shape VLFS with edge facing approaching wave

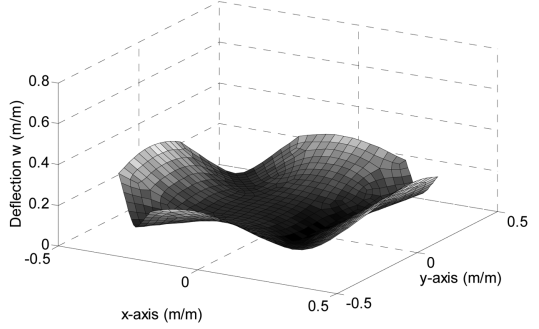

(e) Octagonal shape VLFS with edge facing approaching wave



(b) Circular VLFS



(d) Octagonal shape VLFS with corner pointing approaching wave



(f) Hexagonal VLFS with corner pointing approaching wave

Fig. 8 Hydroelastic response of polygonal VLFS under $\alpha=0.3$

herein. It was found that the hydroelastic response of the longish VLFS under a head sea wave could be effectively reduced by altering the fore- and aft-ends of the VLFS. The elliptical VLFS was found to be most effective in mitigating the hydroelastic response of the longish VLFS due to 
its streamline body profile. Among the various polygonal fore-ends considered, the triangular foreend was found to be the most effective in reducing the hydroelastic response because the incident wave energy is easily scattered away due to the elliptic shape of the floating structure. Note that a greater scattering wave is proportionate to a greater hydrodynamic damping. This phenomenon is being investigated by Kim et al. (2005a). However, the effectiveness of the elliptical and polygonal fore-ends diminished when the longish VLFS is subjected to longer wave lengths $(\alpha>0.3)$ as the responses is dominated by inertia forces when $\alpha$ increases.

For VLFS with various shapes confined in a same square boundary, it was found that the VLFS with circular shape or polygonal shape has a smaller hydroelastic response as compared to a square VLFS. The reduction of the hydroelastic response by using polygonal shapes is significant for higher wavelengths. This is because the hydroelastic response of polygonal shape or circular shape is governed by bending action in both the $x$ - and $y$ - directions. By orientating the square or hexagonal shape VLFS so that the one of the corner/vertex is pointing to the approaching wave, it was found that the hydroelastic response could be reduced significantly as compare to their counterparts that are orientated with the edge facing the approaching wave. This is because the pointed vertex allow for a better flow across the floating body. Hence, the VLFS with a longer edge facing the approaching wave were found to experience higher hydroelastic deformation.

In sum, it was found that the hydroelastic response of VLFS could be reduced by altering the conventional square or rectangular shaped VLFS. The findings reported herein would be useful information to offshore and marine engineers working on arbitrary shaped VLFS placed in a channel or a river.

\section{References}

Brebbia, C.A. (1984), Boundary element techniques: Theory and applications in engineering, Springer-Verlag, New York, USA.

Eatock Taylor, R. and Waite, J.B. (1978), "The dynamic of offshore structures evaluated by boundary integral techniques", Int. J. Numer. Meth. Eng., 13(1), 73-92.

Faltinsen, O.M. (1990), Sea loads on ships and offshore structure, Cambridge University Press, New York, USA.

Gao, R.P., Tay, Z.Y., Wang, C.M. and Koh, C.G. (2011), "Hydroelastic response of very large floating structure with a flexible line conenction", Ocean Eng. (In Press, Corrected Proof).

Khabakhpasheva, T.I. and Korobkin, A.A. (2002), "Hydroelastic behavior of compound floating plate in waves", J. Eng. Math., 44(1), 21-40.

Kim, B.W., Hong, S.Y., Kyoung, J.H. and Choo, Y.S. (2005a), "Year. investigation on wave reduction performances of floating hinge-linked breakwater", Proceedings of the 2nd Joint Japan/Korea Workshop on Marine Environmental Engineering, Fukuoka, Japan.

Kim, B.W., Kyoung, J.H., Hong, S.Y. and Cho, S.K. (2005b), "Investigation of the effect of stiffness distribution and structure shape on hydroelastic response of very large floating structures", Proceedings of the 15th International Offshore and Polar Engineering Conference, Seoul, Korea.

Liew, K.M., Wang, C.M. and Xiang, Y. (1998), Vibration of mindlin plates: Programming the p-version ritz method, Elsevier, Oxford, UK.

Linton, C.M. (1999), "Rapidly convergent representations for Green's functions for Laplace's equation", Proceedings of the Royal Society A: Mathematical, Physical and Engineering Science 455(1985).

Masanobu, S., Kato, S., Maeda, K. and Namba, Y. (2003), "Response of Mega-Float equipped with novel wave energy absorber", Proceedings of the 22nd International Conference on Offshore Mechancis and Arctic Engineering, Cancun, Mexico.

Newman, J.N. (1994), "Wave effect on deformable bodies", Appl. Ocean Res., 16(1), 47-59. 
Ohta, H., Torii, T., Hayashi, N., Watanabe, E., Utsunomiya, T., Sekita, K. and Sunahara, K. (1999), "Effect of attachment of a horizontal/vertical plate on the wave response of a VLFS", Proceedings of the $3^{\text {rd }}$ International Workshop on Very Large Floating Structure, University of Hawaii at Manao, Honolulu, HI.

Pinkster, J.A. (1997), "The effect of air-cushion under floating offshore structures", Proceeedings of the 8th International Conference on the Behavior of Offshore Structure, Delf, The Netherlands.

Sarpkaya, T. and Isaacson, M. (1981), Mechanic of wave forces on offshore structures, Van Nostrand Reinhold Co., New York, USA.

Suzuki, H. (2005), "Overview of megafloat: Concept, design criteria, analysis, and design”, Marine Struct.,18(2), $111-132$

Tay, Z.Y., Wang, C.M. and Utsunomiya, T. (2009), "Hydroelastic responses of interactions of floating fuel stroage modules placed side-by-side with floating breakwaters", Marine Struct., 22(3), 633-658.

Wang, C.M., Gao, R.P. and Koh, C.G. (2012), "Reducing hydroelastic response of very large floating structure using flexible line connector and gill cells", International journal of Structure Stability and Dynamic (IJSSD) Symposium, Nanjing, China, (Submitted).

Wang, C.M., Riyansyah, M. and Choo, Y.S. (2009), "Reducing hydroelastic response of interconnected floating beams using semi-rigid connections", Proceedings of the 28th International Conference on Ocean, Offshore and Arctic Engineering, Honolulu, Hawaii, USA.

Wang, C.M., Tay, Z.Y., Takagi, K. and Utsunomiya, T. (2010), "Literature review of methods for mitigating hydroelastic response of VLFS under wave action", Appl. Mech. Rev., 63(3).

Wang, C.M., Watanabe, E. and Utsunomiya, T. (2008), Very large floating structure, Taylor and Francis, Routledge, UK. 